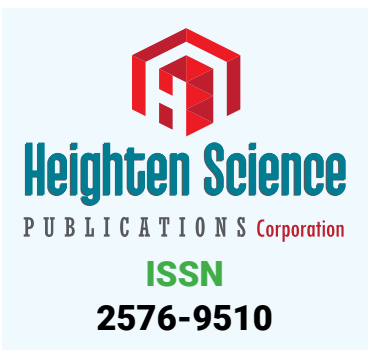

*Address for Correspondence: Williams DL, Queen's Veterinary School Hospital, Department of Veterinary Medicine, University of Cambridge, Madingley Road, Cambridge CB3 OES, UK, Tel: 07939074682; Email: dlw33@cam.ac.uk; doctordlwilliams@aol.com

Submitted: 18 October 2017

Approved: 27 October 2017

Published: 30 October 2017

Copyright: @ 2017 Williams DL, et al. This is an open access article distributed under the Creative Commons Attribution License, which permits unrestricted use, distribution, and reproduction in any medium, provided the original work is properly cited.
Research Article

\section{Ocular surface Rose Bengal staining in normal dogs and dogs with Keratoconjunctivitis Sicca: Preliminary findings}

\author{
Williams DL* and Griffiths A \\ Queen's Veterinary School Hospital, Department of Veterinary Medicine, University of \\ Cambridge, Madingley Road, Cambridge CB3 OES, UK
}

\section{Abstract}

Dry eye or keratoconjunctivitis sicca, is commonly seen in the dog. Veterinary ophthalmologists diagnose this aqueous tear deficiency using the Schirmer tear test (STT), but this measures tear production and does not indicate ocular surface pathology. The vital dye Rose Bengal is commonly used in the diagnosis of dry eye in human patients but until now has not been reported in veterinary patients. Here we corelate the degree of Rose Bengal staining with the STT value and find a reasonable association between dye staining of the ocular surface and tear production, although clearly other factors are also important in the genesis of ocular surface damage in dry eye.

\section{Introduction}

Keratoconjunctivitis sicca (KCS) or dry eye in the dog is a common uncomfortable and potentially blinding condition [1] most frequently diagnosed by use of the Schirmer tear test (STT), first developed by Otto Schirmer for quantification of tear production in people in 1903 [2] and modified for use in the dog by Gelatt in 1975 [3]. A tear production of less than $15 \mathrm{~mm} / \mathrm{min}$ of tear strip wetting is generally considered to diagnose canine KCS. In human patients the STT is considered a less valuable test and a panoply of other diagnostic methods are used from quantification of tear film breakup time through measurement of tear film osmolarity to evaluation of ocular surface pathology through use of vital dye staining using Rose Bengal or Lissamine Green stains. In 1933 Sjogren described the staining pattern observed in patients with KCS and he speculated that the dye was staining normal but desquamated corneal epithelial squames [4]. Passmore continued this theory in his 1955 review [5] and it was not until Norn's work in the 1960s that the view changed to consider that Rose Bengal was staining dead and degenerate epithelial cells [6-8]. Twenty years later Scheffer Tseng discovered that the dye stained a range of healthy cells in culture, leading him to ask whether healthy corneal cells were stained and if so, why healthy cornea was not stained. His groups work [9] showed that the dye did stain cultured corneal epithelium and indeed was mildly toxic to migrating cells. Given that bovine serum albumen and carboxymethylcellulose block this staining, Tseng suggests that mucus on the ocular surface does the same thing and that it is a deficiency of mucus in KCS that renders affected corneas liable to take up stain. Whatever the mechanism of action, Rose Bengal is a widely used stain in human eyes with KCS and it seems only reasonable to investigate if it has a part to play in the diagnosis of tear film deficiency in the dog. Here we present preliminary findings on Rose Bengal staining of the ocular surface of dogs with normal tear production and eyes with aqueous tear film deficiency.

How to cite this article: Williams DL, Griffiths A. Ocular surface Rose Bengal staining in normal dogs and dogs with Keratoconjunctivitis Sicca: Preliminary findings. Insights Vet Sci. 2017; 1: 042-046. https://doi.org/10.29328/journal.ivs.1001005 


\section{Materials and Methods}

All dogs in the study were presented for assessment and treatment of ophthalmological conditions at the Queens' Veterinary School Hospital, Department of Veterinary Medicine, University of Cambridge from September 2014 to June 2015. Following a full ophthalmological examination including direct and indirect ophthalmoscopy (Practitioner direct ophthalmoscope and Vantage indirect ophthalmoscope, both Keeler, Windsor UK), STTs were performed using one batch of tear strips (EagleVision, Monterey, California) and the value of tear strip wetting after one minute was recorded. After thirty minutes a Rose Bengal strip was used with one drop of topical $10 \%$ sterile saline to apply dye to the surface of each eye. Photographs were taken immediately using a Nikon Coolpix 4500 digital camera held at $10 \mathrm{~cm}$ from the eye, so that the degree of staining could be assessed at a later date with assessors blinded to the clinical status of the dog. Staining was categorised by the scale shown in table 1 . Analysis of images was undertaken by ten individuals who were shown the images with no signalment data and asked to rate each one according to the scale in table 1. Eight were final year veterinary students and two were adults in nonveterinary professions. None had any prior experience with Rose Bengal usage in either humans or animals.

The study was evaluated and accepted by the Ethics and Welfare committee of the Department of Veterinary Medicine, University of Cambridge. All owners gave fully informed consent for their animal's inclusion in the study. In humans Rose Bengal is noted to cause ocular irritation in some patients. In such cases in the current study it was decided that topical and, if necessary systemic pain relief would be given in such cases but ocular irritation was not noted in any of the animals involved.

\section{Results}

Ten adult dogs of varying breed (Bernese Mountain Dog (1), Lurcher (1), Greyhound (2), Labrador (3), Golden Retriever (2), Boxer (1)) aged between 3 and 9 years of age were used as a control population. These dogs had normal STT results (all over $15 \mathrm{~mm} /$ min with a range $15-22 \mathrm{~mm} / \mathrm{min}$ and mean \pm standard deviation $18.6 \pm 1.7$ ) and no signs of corneal pathology nor ocular irritation. No staining with Rose Bengal was evident in the 20 eyes of the 10 control dogs (scores of 0 were given for all twenty eyes by all observers).

The KCS group comprised 20 adult dogs with 36 eyes with STT $<15 \mathrm{~mm} / \mathrm{min}$ )) all diagnosed with to uni-ocular or bilateral KCS. Only eyes with KCS were included in the study. The breeds were West Highland White Terriers (6), Cavalier King Charles Spaniels (4), English Cocker Spaniel (3), American Bulldog (2), Lhasa Apso (2), Pug (1), Mastiff (1) and crossbreed (1). The ages of these animals ranged from 6 to 12 years, with a median of 8 years and a mean of 8.2 years.

In the KCS group, the mean STT value was $6.3 \pm 5.2 \mathrm{~mm} / \mathrm{min}$ (range $0-14 \mathrm{~mm} / \mathrm{min}$ ) and mean Rose Bengal score was 3.05 \pm 3.3 (range 0.9-5) following averaging of the ten independent scorers. Mean responses between scorers for each image varied up to a maximum of 1 point with an average variation of 0.8 points and a strong correlation between scores between observers for the same eye with a minimum $R$ value of 0.70 and maximum of 0.93 . Ocular surface staining in dogs with dry eye are shown in figure 1.

\begin{tabular}{|c|c|}
\hline \multicolumn{2}{|c|}{ Table 1: Gradation of degree of staining. } \\
\hline 0 & Less than $20 \%$ of visible surface stained \\
\hline 1 & Between 20 and $40 \%$ of visible surface stained \\
\hline 2 & Between 40 and $60 \%$ of visible surface stained \\
\hline 3 & Between 60 and $80 \%$ of visible surface stained \\
\hline 4 & Greater than $80 \%$ of visible surface stained \\
\hline 5 & \\
\hline
\end{tabular}




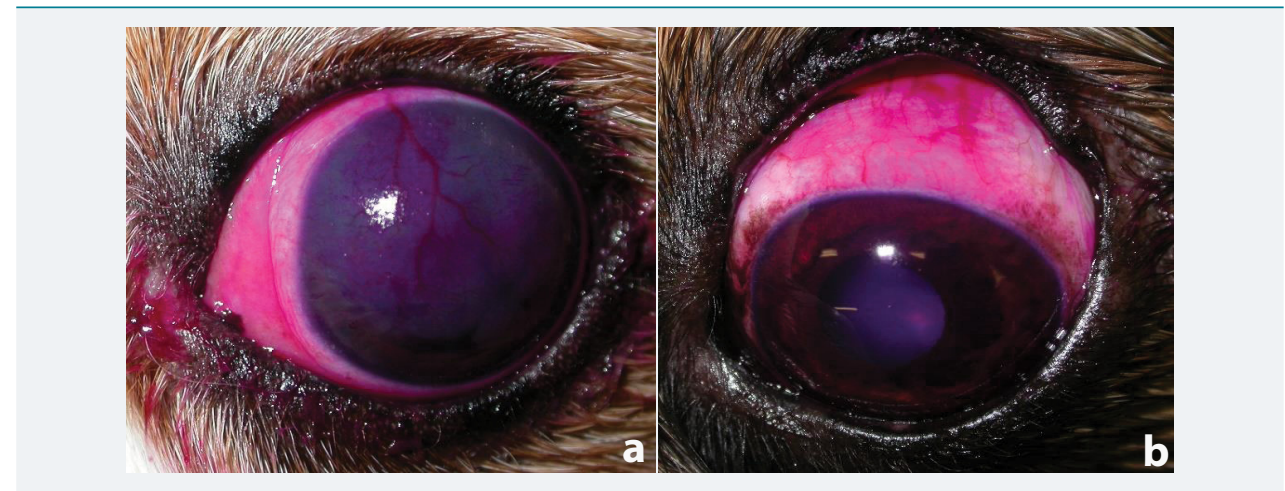

Figure 1: Rose Bengal staining of conjunctiva and cornea in two dogs with KCS.

a) 8 year old Cavalier King Charles spaniel with STT of $4 \mathrm{~mm} / \mathrm{min}$ and staining both of conjunctiva and cornea together with corneal haze and neovascularisation. b) 7 year old Cross-bred dog with STT of $9 \mathrm{~mm} / \mathrm{min}$ and staining confined to the conjunctiva.

It can be seen from Figure 1 that the spread of results appears broader at the lowest STT results. For this reason a further analysis was undertaken to separate groups into those with STTs at $5 \mathrm{~mm} / \mathrm{min}$ and above, and those below. For STT values below $5 \mathrm{~mm} / \mathrm{min}$ analysis showed a moderate negative correlation (Pearson's correlation coefficient, $\left.\mathrm{R}:-0.62, \mathrm{R}^{2}: 0.39, \mathrm{P}=0.00013\right)$. As can be appreciated from graph in figure1, the spread of results appears 5, R:-0.068 (not significant at $\mathrm{P}<0.05$ ), whereas for those at 5 or above, $\mathrm{R}:-0.82, \mathrm{P}=0.000026$ ). The sample size was too small to examine whether significant differences in Rose Bengal staining were noted between different breeds or age groups was found and such work awaits further research.

\section{Discussion}

For human patients with dry eye the Schirmer tear test is generally considered not to be particularly valuable and because of this a number of other tests are used. In veterinary ophthalmology on the other hand the ease of use and simplicity of the STT has led to its widespread use in diagnosis canine KCS. Having said that the ocular pathology seen in canine dry eye may not perfectly correlate with tear wetting of the Schirmer tear test strip and other diagnostic tests may be valuable in addition to the STT. We have recently detailed changes in osmolarity of the tear film [10] which in the human patient are considered a key factor causing ocular surface pathology in aqueous deficiency dry eye. The Rose Bengal stain may be valuable in documenting this corneal and conjunctival pathology directly. Over the years of its use there has been some debate over exactly what Rose Bengal stains. It used to be thought that the dye bound to dead or devitalised cells but now the consensus is that the dye binds to tissue that is devoid of mucus covering [9,11]. Whatever the exact mechanism, the stain evaluates ocular surface pathology rather than merely tear production as does the STT. For that reason the Rose Bengal stain should be a valuable addition to the diagnostic tests used in canine dry eye. Overall there is a correlation between Rose Bengal staining and the ST result, as shown statistically above and in the graph in figure 2 . The correlation however, is not perfect. No eyes with STT below $15 \mathrm{~mm} / \mathrm{min}$ had staining scores of zero and no dogs with STT above $15 \mathrm{~mm} / \mathrm{min}$ stained with Rose Bengal. However there were eyes with a low STT which still had mean scores of less than 3 and, to a lesser extent, dogs with STT values of between 5 and $10 \mathrm{~mm} / \mathrm{min}$ which showed some degree of staining, so the correlation is not exact.

There are potential limitations in this study. A small number of dogs were evaluated, hence our acknowledgement in the title of this study that these are preliminary findings. We obtained dye staining scores from individuals without expert knowledge of veterinary ophthalmology to simulate the situation in general veterinary practice but a more strictly quantitative approach could have been to perform image analysis on the photographs to determine exactly the percentage of ocular surface stained, 


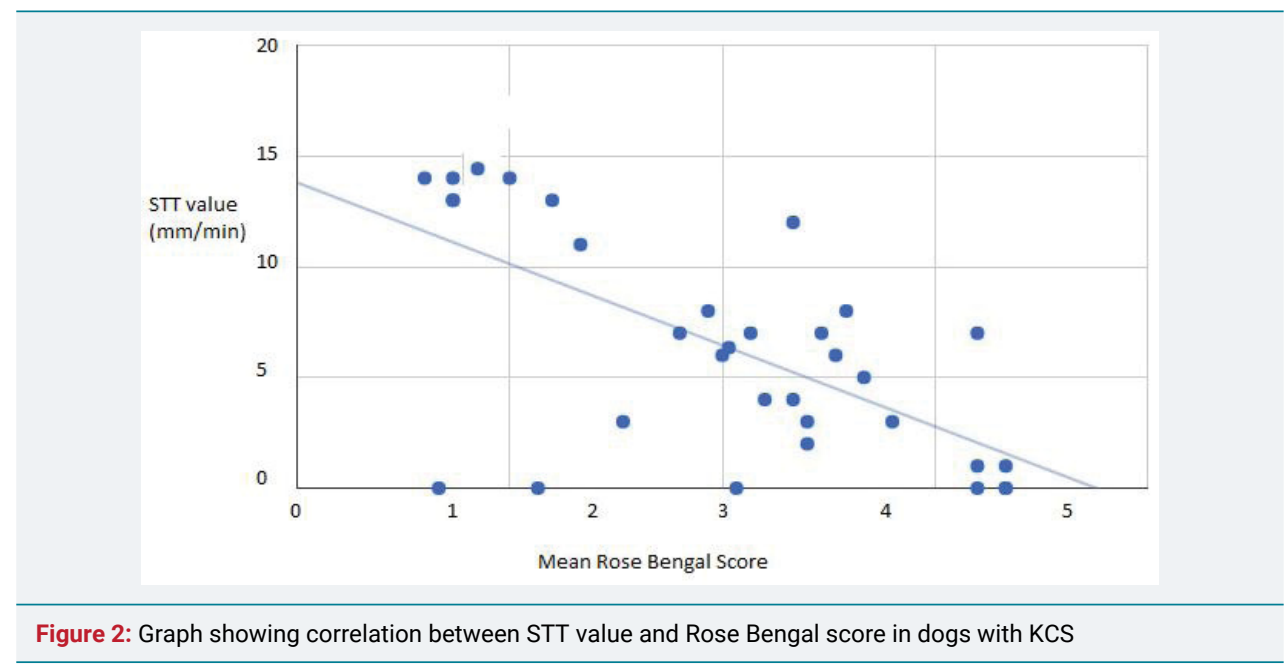

as was previously undertaken in a study of treatment of a chronic canine keratitis [12]. This however highlights further issues with the evaluation of ocular surface staining exemplified by the two images shown in figure 1. Figure 1a shows staining of conjunctiva and cornea in a severe case of KCS. The corneal stain is stippled and defining the exact extent of staining is difficult. Figure $1 \mathrm{~b}$ shows an eye with less severe KCS where there is no corneal staining but substantial conjunctival staining. We did not instruct the image assessors whether to include both surfaces in their assessment or to attempt to include the intensity of staining in their final score. Equally each image shows one view of the eye while evaluation of the eye in the living patient where a more accurate evaluation of the entire ocular surface would be possible. Even given these limitations the assessors' results were similar.

\section{Conclusion}

Based on the findings from this small study we would recommend the use of Rose Bengal staining as a valuable additional technique for evaluation of the eye in cases of keratoconjunctivitis sicca. Further work assessing the technique in a larger number of dogs with keratoconjunctivitis sicca would be valuable as might investigations of another vital dye Lissamine Green used in the clinical assessment of human dry eye.

\section{References}

1. Williams DL. Immunopathogenesis of keratoconjunctivitis sicca in the dog. Vet Clin North Am Small Anim Pract. 2008; 38: 251-268. Ref.: https://goo.gl/rJZUPb

2. Schirmer 0. Studien zur physiologie und pathologie der tränenabsonderung und tränenabfuhr. Graefe's Archive for Clinical and Experimental Ophthalmology. 1903; 56: 197-291. Ref.: https://goo.gl/W458V6

3. Gelatt KN, Peiffer RL Jr, Erickson JL, Gum GG. Evaluation of tear formation in the dog, using a modification of the Schirmer tear test. J Am Vet Med Assoc. 1975; 166: 368-370. Ref.: https://goo.gl/aokwK2

4. Sjogren H. Zur kenntnis der keratoconjunctivitis sicca (keratitis filiformis bei hypofunktiondder tranendrusen. Acta Ophthalmol. 1933; 86: 36-39. Ref.: https://goo.gl/WuTzG8

5. Passmore JW, King JH Jr. Vital staining of the conjunctiva and cirnea: a review of the literature and critical study of certain dyes. Arch Ophthalmol. 1955; 153: 568-574. Ref.: https://goo.gl/DCqCCX

6. Norn MS. Specific double vital staining of the cornea and conjunctiva with Rose Bengal and Alcian Blue. Acta Ophthalmol (Copenh). 1964; 42: 84-96. Ref.: https://goo.gl/bSfUbB

7. Norn MS. Vital staining of the cornea and conjunctiva; with a mixture of fluorescein and rose bengal. Am J Ophthalmol. 1967; 64: 1078-1080. Ref.: https://goo.gl/wYVdSM

8. Norn MS. Rose Bengal vital staining. Staining of cornea and conjunctiva by 10 percent rose bengal, compared with 1 percent. Acta Ophthalmol (Copenh). 1970; 48: 546-559. Ref.: https://goo.gl/axJAVQ 
9. Feenstra RP, Tseng SC. What is actually stained by rose bengal? Arch Ophthalmol. 1992; 110: $984-$ 993. Ref.: https://goo.gl/YJ7t6Q

10. Williams DL, Buckingham A. Measurement of tear osmolarity in the canine eye: a new diagnostic tool for canine keratoconjunctivitis sicca? J Vet Sci. 2017; 3: 8-12. Ref.: https://goo.gl/3MpFks

11. Pflugfelder SC, Tseng SC, Yoshino K, Monroy D, Felix C, et al. Correlation of goblet cell density and mucosal epithelial membrane mucin expression with rose bengal staining in patients with ocular irritation. Ophthalmology. 1997; 104: 223-235. Ref.: https://goo.gl/s5kxZE

12. Williams DL, Hoey AJ, Smitherman P. Comparison of topical cyclosporin and dexamethasone for the treatment of chronic superficial keratitis in dogs. Vet Rec. 1995; 137: 635-639. Ref.: https://goo.gl/1E4WGk 